\title{
Development of a Double-Pole Double-Throw Radio Frequency Micro Electro-Mechanical Systems Switch using an 'S' Shaped Pivot
}

\author{
Mohammed A. Al-Amin, ${ }^{\text {a* }}$ Sufian Yousef, ${ }^{a}$ Barry Morris, ${ }^{a}$ Hassan Shirvani, ${ }^{a}$ Michael Cole a \\ ${ }^{a}$ Anglia Ruskin University, Science and Technology, Engineering and Built Environment, Bishop Hall Lane, \\ Chelmsford, Essex, United Kingdom, CM1 1SQ
}

\begin{abstract}
This paper investigates the design of a novel
pivot for a seesaw, RF MEMS, double-pole double-throw (DPDT) switch, which has been developed to operate within mobile communication systems and devices. The pivot employs a unique ' $S$ ' structure at the nano scale, in the form of a, which helps to keep von-Mises stresses below $21 \mathrm{MPa}$. The pivot requires less pulling force than similar designs due to its flexibility which allows the beam and contacts a greater space of separation while the switch is off. This in turn results in improved contact isolation of greater than $-77 \mathrm{~dB}$ at $5 \mathrm{GHz}$. The RF MEMS switch is an improvement over the previously published paper (Al-Amin et al. in International symposium on microelectronics, vol 2013, no 1, pp 000831-000835, 2013. doi:10.1109/ ECS.2014.6892558), since the pulling force of the electrostatic plates can be generated with a voltage which is greatly reduced from 14 to $8 \mathrm{~V}$ using the same electrostatic plate area size. The switch is a progression from SPST and DPDT seesaw switching since it provides improved flexibility over the previously described devices.
\end{abstract}

The switch is a progression from SPST and DPDT seesaw switching since it provides improved flexibility over the previously described devices.

With the redesign of the pivot the switch attains a greater 'air-gap' between the contacts when open-circuited which therefore allows for improved isolation during the off-state.

Keywords: DPDT, Electromagnetic Analysis, Pivot, RF MEMS, Seesaw.

*Mohammed A. Al-Amin, E-mail: mohammed.al-amin@student.anglia.ac.uk

\section{Introduction}

RF MEMS (Radio Frequency Micro Electro- Mechanical Systems) are small micro components, used in micro-devices or microchips, which use sub-millimeter movements in order to create a physical contact or disconnect. RF MEMS require little drive current since they are voltage driven, which increases battery time. MEMS technology is widely used in sensors for many different applications but their use in RF technologies is relatively new compared to solid state semiconductor switches. 
This paper presents a concept to the seesaw pivot allowing greater flexibility, with reduced vonmises stress, which in turn increases reliability ${ }^{1}$. This novel ' $\mathrm{S}$ ' shaped pivot provide the beam with more flexibility over a straight $\operatorname{pivot}^{3}$ due to a reduction in elastic recovery. This allows the airgap to be increased between contacts for greater isolation.

This paper focuses on the application of RF MEMS switching specifically for use within mobile phone devices. However, the technology is equally applicable in other communications devices including satellite communications systems and mobile base-stations.

The Seesaw, RF MEMS switch described here adopts similar design characteristics to those in other papers such as J.M Cabral \& A. S. Holmes ${ }^{7}$ and Jongseok Kim et al $^{5}$. These studies simulated and designed a Single-Pole Single-Throw (SPST) switch with similar voltages to the original Seesaw RF MEMS DPDT Switch ${ }^{3}$. However, the designs were limited in the number of switching contacts. This paper and the previous paper $^{3}$ addresses this limitation with additional contacts to the beam structure, to provide increased functionality within the limited space envelope.

The principle operation of RF MEMS switching relies on electrostatic forces to the close contacts, described in Equation 1. The force $(\mathrm{F})$ between electrostatic plates is described by:

$$
F=\frac{V^{2} \varepsilon_{\mathrm{o}} A}{2 d^{2}}
$$


Where:

$\mathrm{V}=$ Supply voltage

$\varepsilon_{0}=$ Permittivity of free space, in a vacuum

$A=$ Area of the electrostatic plate

$\mathrm{d}=$ Distance between electrostatic plates

$\mathrm{F}=$ Force between two electrostatic plates

Equation 1 does not take into account fringe capacitance which is the capacitance due to the electric fields produced at the edges of the electrostatic plates. This can be represented in the equation by adding a constant $\mathrm{C}$ to provide an accurate result. However, the simulation software Intellisuite takes fringe capacitance into account in its simulation.

The improved design, which incorporates a new copper S-shaped pivot, with a thickness of 0.04 $\mu \mathrm{m}$ (Fig. 5), achieved sufficient pulling force with an activation voltage of only 8 volts.

The new pivot is $0.0047 \mu \mathrm{m}$ thinner than the straight pivot described in the previous paper ${ }^{3}$. The force can be increased by three methods. The first is to increase the area (A) of the electro-static plates; the second is to increase the voltage (V) across the plates and the third is to decrease the distance (d) between them. To ensure that the upper contacts at one side of the beam and the lower contacts at the other side are closed at the same time the beam needs to be flexible. Without this flexibility, early contact on one side of the beam could prevent the opposite side from making 
contact due to errors in symmetry. A flexible beam would overcome this potential problem in order to provide the maximum contact surface area, thus a lower resistance ${ }^{4}$.

\section{Design}

The design, shown in Fig. 1, was chosen for its multiple layers which provides flexibility, reduces the angle of bend and provides a broad distribution of stress across the pivot. Having curved edges helps to reduce the build-up of stress which decreases von-mises forces at the pivot over the yield strength and therefore reduces the risk of deformation. The strengthened curved edges help to distribute the stress along the pivot, this curving of the edges represents the look of an ' $\mathrm{S}$ ' shape.

In order to create this complex ' $S$ ' shape pivot, the orientation of the switch would need to be on its side, to simplify the manufacturing process. This provides two advantages; the first is to save space due to the height of the switch being shorter than its width. The second is that the shape of the pivot can now be easily applied though a layer by layer approach, suitable for MEMS fabrication.

The original and the ' $\mathrm{S}$ ' shape pivot design has had a few changes. Firstly, the materials used for the beam and electrostatic plates have been changed from Aluminum $(\mathrm{Al})$ to Copper $(\mathrm{Cu})$; this is to provide uniform use of materials and reduce complexity of the design as there are less types of materials required. Also, the 'Air Gap' has been increased; this provides improved isolation of the contacts during its 'Off-State' 


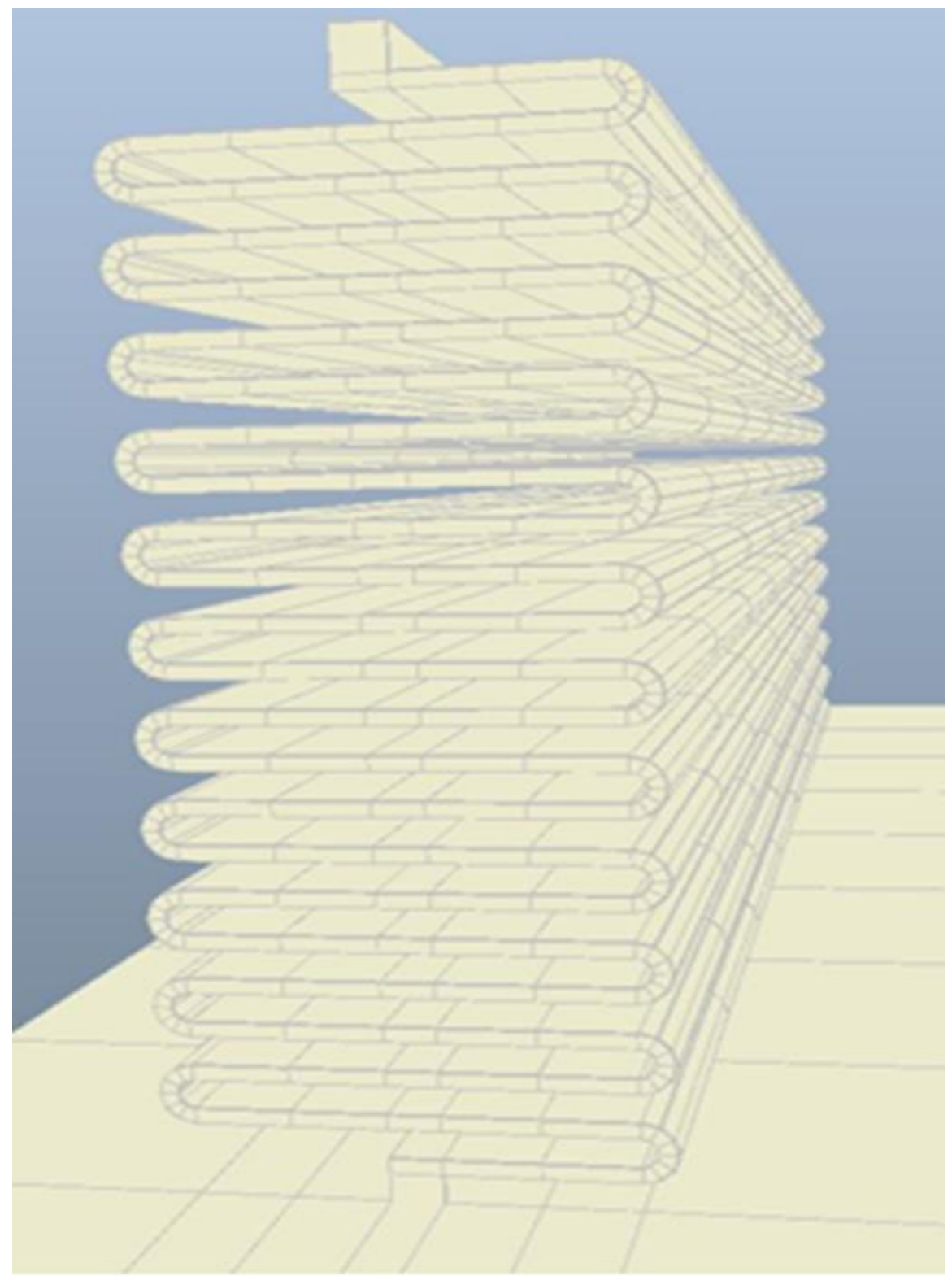

Fig. 1 'S' shaped pivot design

Table 1 Material information of the 'S' shaped pivot seesaw switch

\begin{tabular}{ll}
\hline Component & Material \\
\hline Pivot & Copper $(\mathrm{Cu})$ \\
Beam & Copper $(\mathrm{Cu})$ \\
Bridge Contacts & Gold $(\mathrm{Au})$ \\
Contacts & Gold $(\mathrm{Au})$ \\
Substrate & Silicon $(\mathrm{Si})$ \\
Electrostatic Plates & Copper $(\mathrm{Cu})$ \\
\hline
\end{tabular}


Table 2 Material information of RF MEMS DPDT seesaw switch from previous paper ${ }^{3}$

\begin{tabular}{ll}
\hline Component & Material \\
\hline Pivot & Copper $(\mathrm{Cu})$ \\
Beam & Aluminum $(\mathrm{Al})$ \\
Bridge Contacts & Gold $(\mathrm{Au})$ \\
Contacts & Gold $(\mathrm{Au})$ \\
Substrate & Silicon $(\mathrm{Si})$ \\
Electrostatic Plates & Aluminum $(\mathrm{Al})$ \\
\hline
\end{tabular}

Table 3 Dimensions of the 'S' shaped RF MEMS pivot DPDT switch and RF MEMS DPDT seesaw switch from previous paper ${ }^{3}$

\begin{tabular}{lll}
\hline Seesaw Dimensions & S Shaped Pivot $(\boldsymbol{\mu m})$ & Original Pivot $(\boldsymbol{\mu m})$ \\
\hline Air Gap & 1 & 2.7 \\
Beam Length & 41 & 41 \\
Beam Height & 4 & 4 \\
Beam Width & 5 & 5 \\
\hline
\end{tabular}

\section{Design Procedure}

The original DPDT seesaw switch was created based on existing SPST seesaw designs with modifications for improvements. These modifications include upper and lower contacts, at each side of the beam, to convert the SPST Seesaw Switch ${ }^{1,5}$ into a DPDT switch operating in two axes. The new design incorporates an S-shaped pivot, which is attached to the beam to provide elastic recovery. This enables the switch to operate in all three axes.

The Intellisense materials database contains standard materials which were used in the design. The original DPDT seesaw design used the materials listed in Table 2. Copper Bulk General was used for the pivot due to its higher Young's Modulus and yield strength compared to Aluminum Bulk General. The elastic recovery of Copper Bulk General is an important property of the pivot in order to enable the 'off-state' for switching to occur'. 
It was later discovered that using a based pivot provides greater flexibility and allows less stress to be placed at the pivot. It was then realized that the design needed to be simplified from the original switch as it was using too many materials which increased complexity and cost to manufacturing.

\section{Methodology}

A suitable length of $42 \mu \mathrm{m}$ was chosen with the microscale $(1 \mu \mathrm{m}$ to $100 \mu \mathrm{m})$. This is large enough to take advantage of the existing fabrication technology and small enough to operate high switching speeds.

When the beam is in the off-state, the air-gap between the beam and fixed contacts is $2.7 \mu \mathrm{m}$ (see Table 3). This is almost triple the distance of the air-gap described in a previous study ${ }^{3}$.

Each pair of electrostatic plates, within the S-shaped pivot, seesaw design, receives alternating, single polarity, activation pulses, which reduces the need for complex, external control circuitry. Elastic recovery is used as the mechanism for achieving an off-state, without activation pulses. The model shown in Fig. 2 is on its side to facilitate the manufacturing process.

One of the advantages of using the RF MEMS seesaw design, incorporating DPDT switching ${ }^{3}$, is that two distinct radio frequencies can be accommodated for simultaneous, MIMO (Multiple-in Multiple-out) communications, via a dual antenna array. This additional functionality is achieved within a footprint area of only $369 \mu \mathrm{m}^{2}$ (i.e. $41 \mu \mathrm{m} \times 9 \mu \mathrm{m}$ ) which facilitates the integration with highly constrained semiconductor designs. 
Using a minimum feature size of $40 \mathrm{~nm}$ or $47.6 \mathrm{~nm}$ would cause fabrication difficulties due to the pivot being the thinnest component of the structure. This creates an extremely high aspect ratio, which cannot be implement using today's technology. However, it is a concept that is provided for future designers to implement when the technology becomes available. The ' $S$ ' shaped seesaw design provides similar advantages during etching processing due to its simple design, as it allows the etching solution to run though the structure without being held in the gaps.

\section{Switching Techniques}

Switching between dual input and output configurations may be implemented using the RF MEMS seesaw design. For example, switching between two RX (receive) and TX (transmit) frequency bands can be achieved.

Simultaneous RX and TX communications may be implemented, at two distinct RF frequency bands, by connecting RF mixers to the contacts. The result of this configuration provides dual RX/TX switching capability at four frequencies.

The seesaw RF MEMS switch is ideally suited to operate with common protocols, such as: Wi-Fi, GSM, Bluetooth, WiMAX, GPS, 3G, 4G and 5G. Since most of these protocols operate at frequencies of less than $5.8 \mathrm{GHz}$, any limitations are due to the quality of the antenna rather than the switching device ${ }^{8}$. 


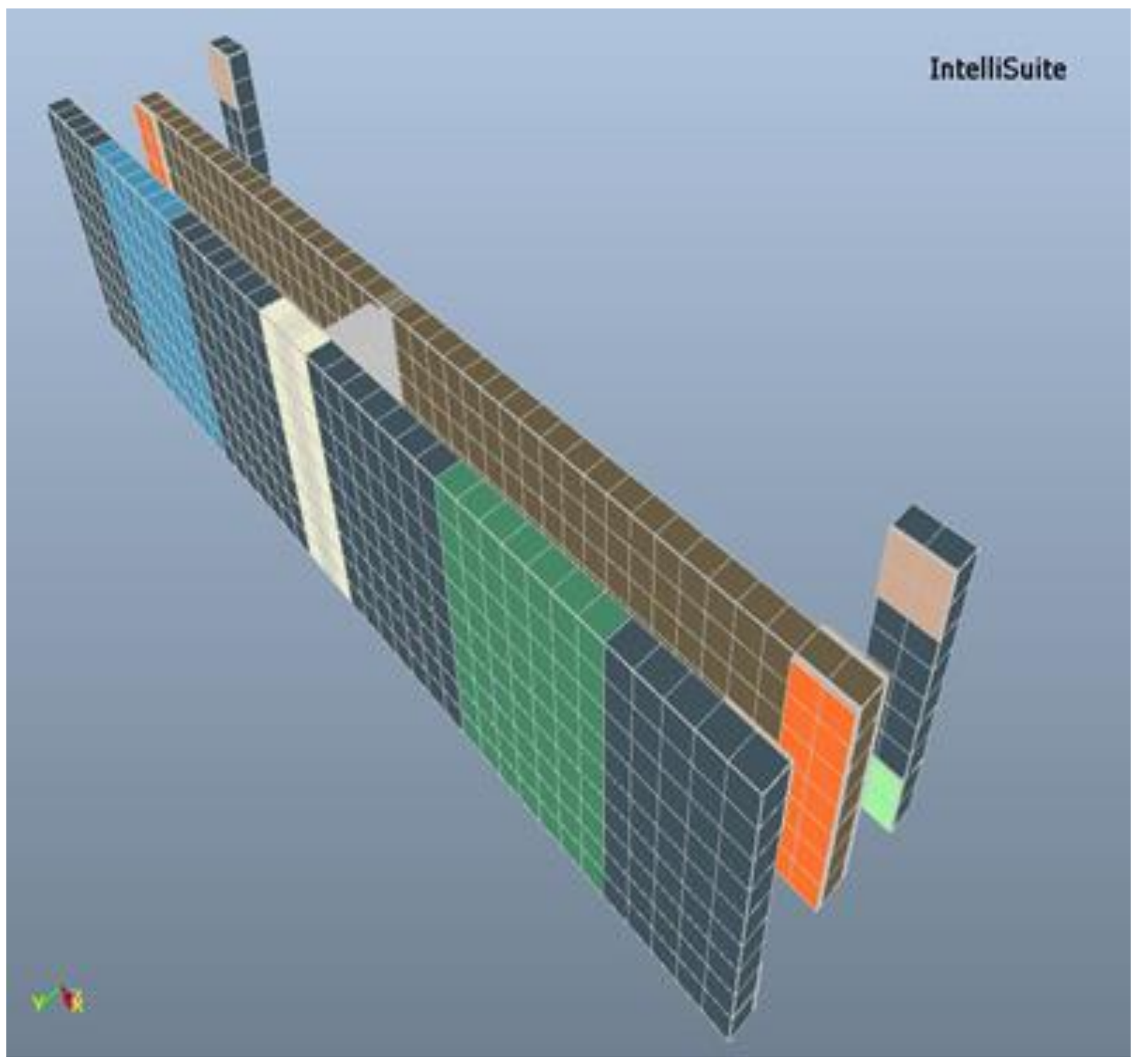

Fig. 2 RF MEMS 'S' shaped pivot, laid on its side for the manufacturing processes 


\section{Testing Parameters Methodology}

Intellisense simulation software was used to test the RF MEMS switch. Fixed pulse widths were supplied to the electrostatic plates under simulation control. The amplitude of these pulses were increased in incremental steps until a sufficient displacement was reached, which enabled the contacts to close. The simulation package could be used to display the results in graphical format and also export the associated data into a Microsoft Excel spread-sheet for numerical analysis ${ }^{9}$.

The design parameters were then transferred to the CST EM (Computer Simulation Technologies Electro-Magnetic) simulation tool where the electro-magnetic characteristics of the switch were observed and recorded.

The results obtained are the Scatter (S) parameters for Isolation and Insertion loss. These results are displayed by the simulation software in graphical format which are displayed in Fig. $3^{10}$. 


\section{$7 \quad$ Results}

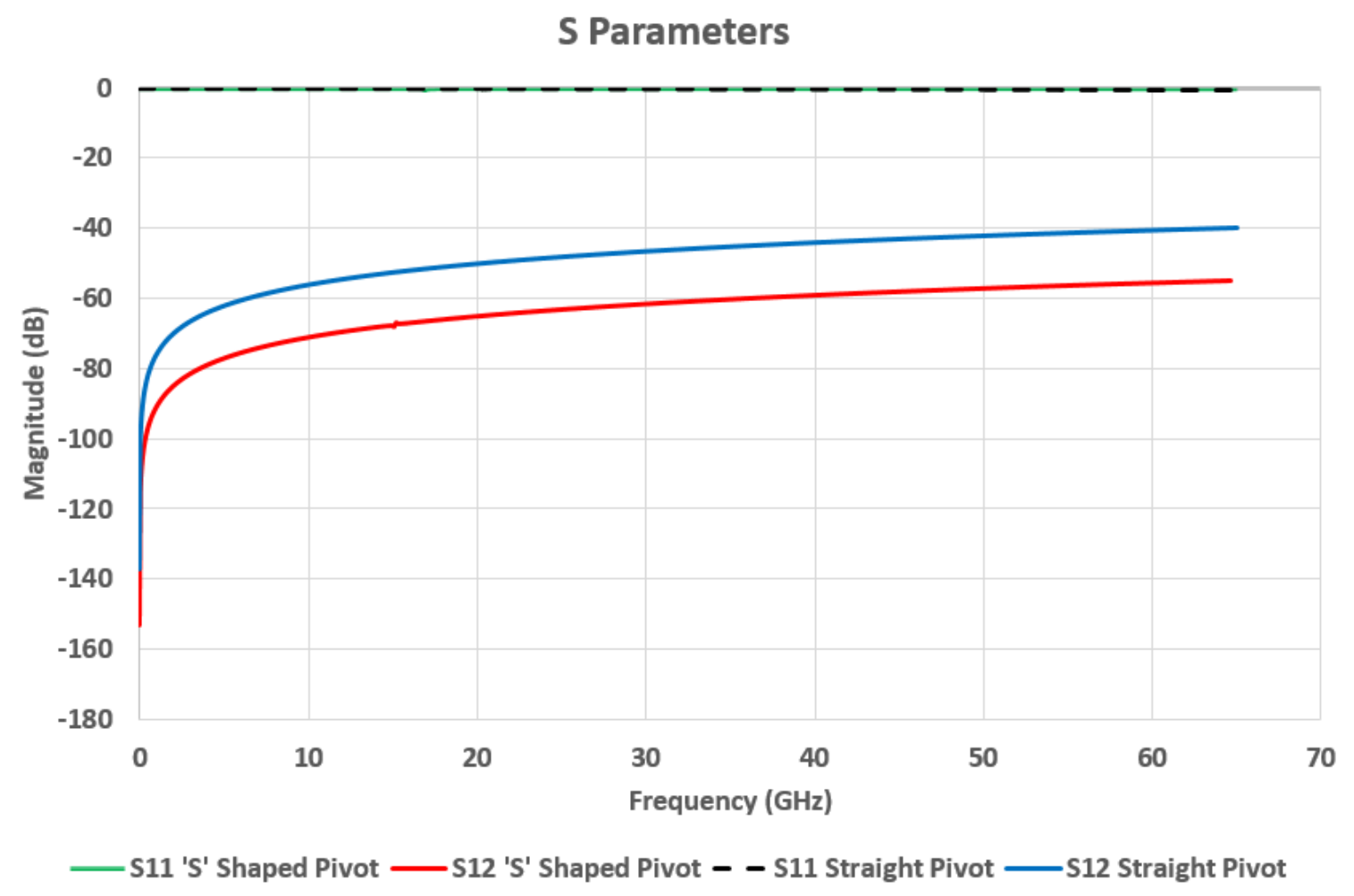

Note: The S11 parameters overlap each other.

Fig. 3 S-Parameters of contacts for the Straight and 'S' Shaped pivot switches

Fig. 3 provides a graphical analysis from CST of the S-parameters for the original DPDT and the 'S' shaped pivot switch. As the switch provides an 'Air-gap' which is almost 3 times greater than the original, it shows an almost $20 \mathrm{~dB}$ improvement in isolation (S12) throughout the frequency range with a reflection coefficient (S11) of 0dB. Previous papers have shown ${ }^{1,5}$ that isolation values range from, typically, $-20 \mathrm{~dB}$ to $-50 \mathrm{~dB}$ within the frequency range from $2 \mathrm{GHz}$ to $2.3 \mathrm{GHz}$, with an air gap between $1 \mu \mathrm{m}$ to $10 \mu \mathrm{m}$. The 'S' shaped seesaw RF MEMS switch shows an -85 $\mathrm{dB}$ to $-84 \mathrm{~dB}$ at $2 \mathrm{GHz}$ to $2.3 \mathrm{GHz}$ respectively, which is an improvement to the typical values specified in the previous sentence due to the distance between contacts and their areas. 


\section{von Mises Stress (MPa)}

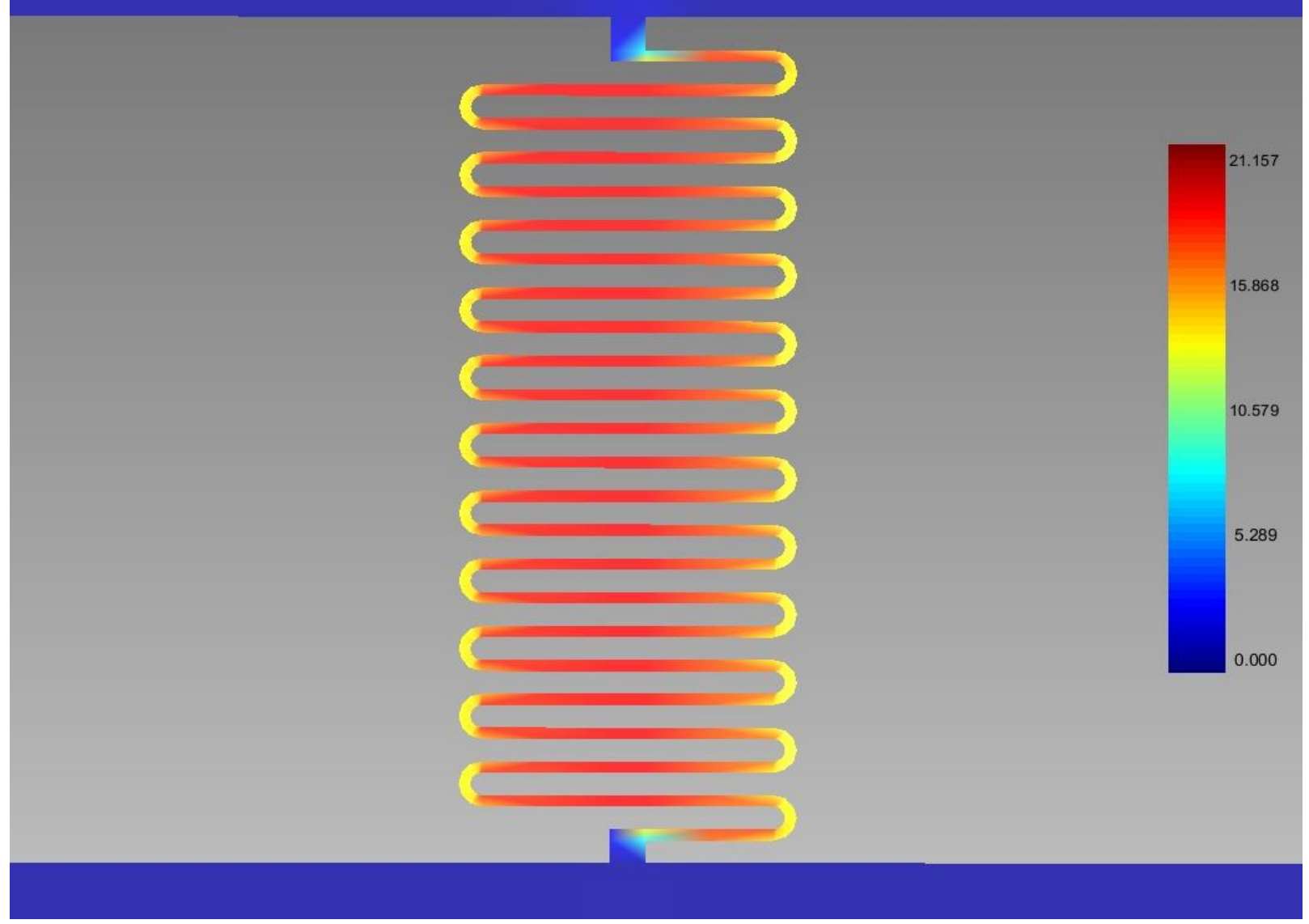

Fig. 4 Color coded von Mises Stress distribution on the 'S' shaped Pivot 


\section{von Mises Stress (MPa)}

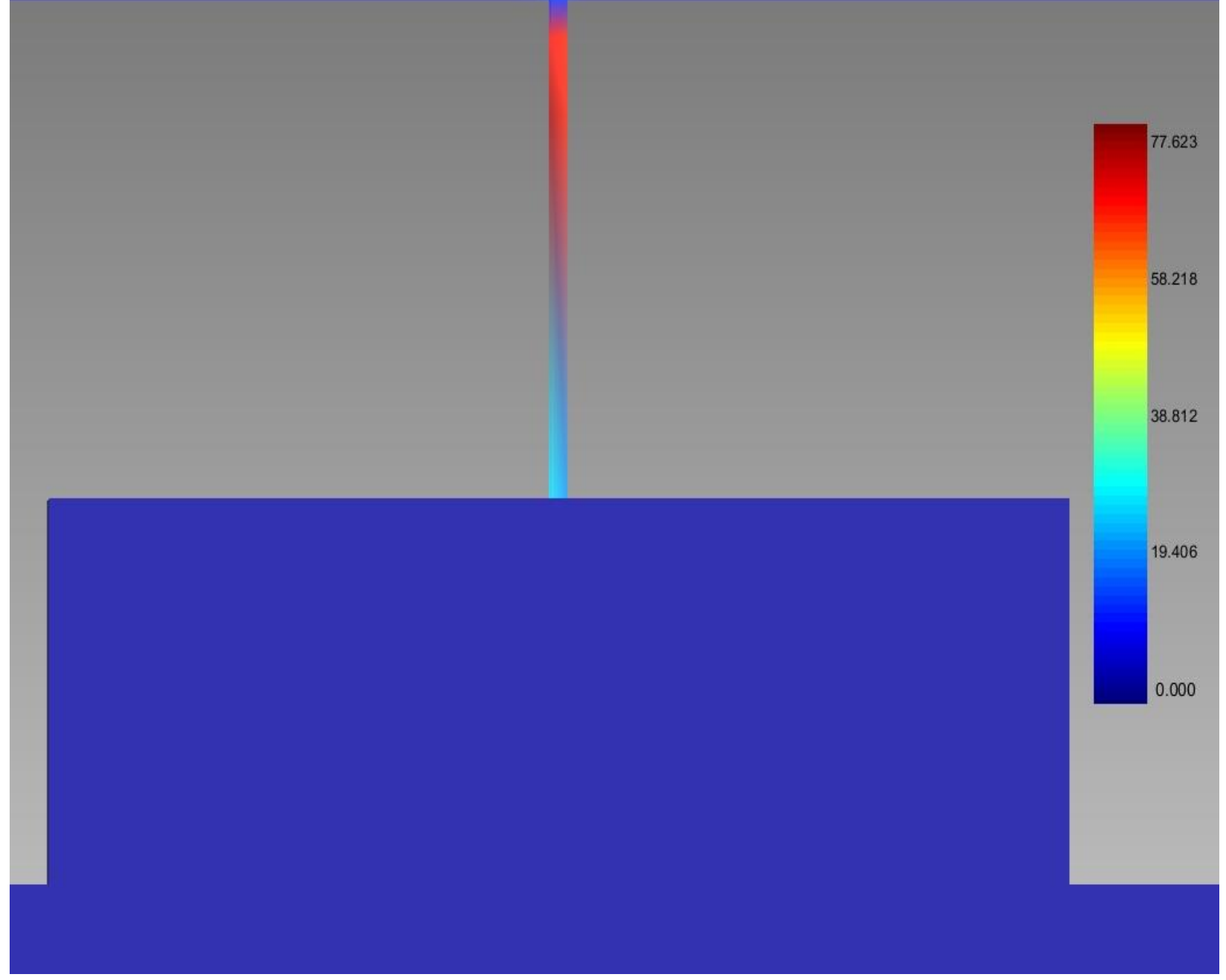

Fig. 5 Color coded von Mises stress of the original DPDT seesaw switch pivot

Comparing Fig. 4 and 5 it can be seen that the von Mises stress on Fig. 4 is distributed across the pivot, compared to Fig. 5, which is focused nearer to the junction of the pivot and the beam. This focused stress causes friction to occur which leads to heat build-up with subsequent fatigue and fracture. The 'S' shaped design, shown in Fig 4, has a maximum stress level of $21 \mathrm{MPa}$, compared to $77 \mathrm{MPa}$ for the design shown in Fig 5. This improvement of $56 \mathrm{MPa}$ is due to the stress being distributed within each link of the 'S' shape. 


\section{Displacement vs Time}

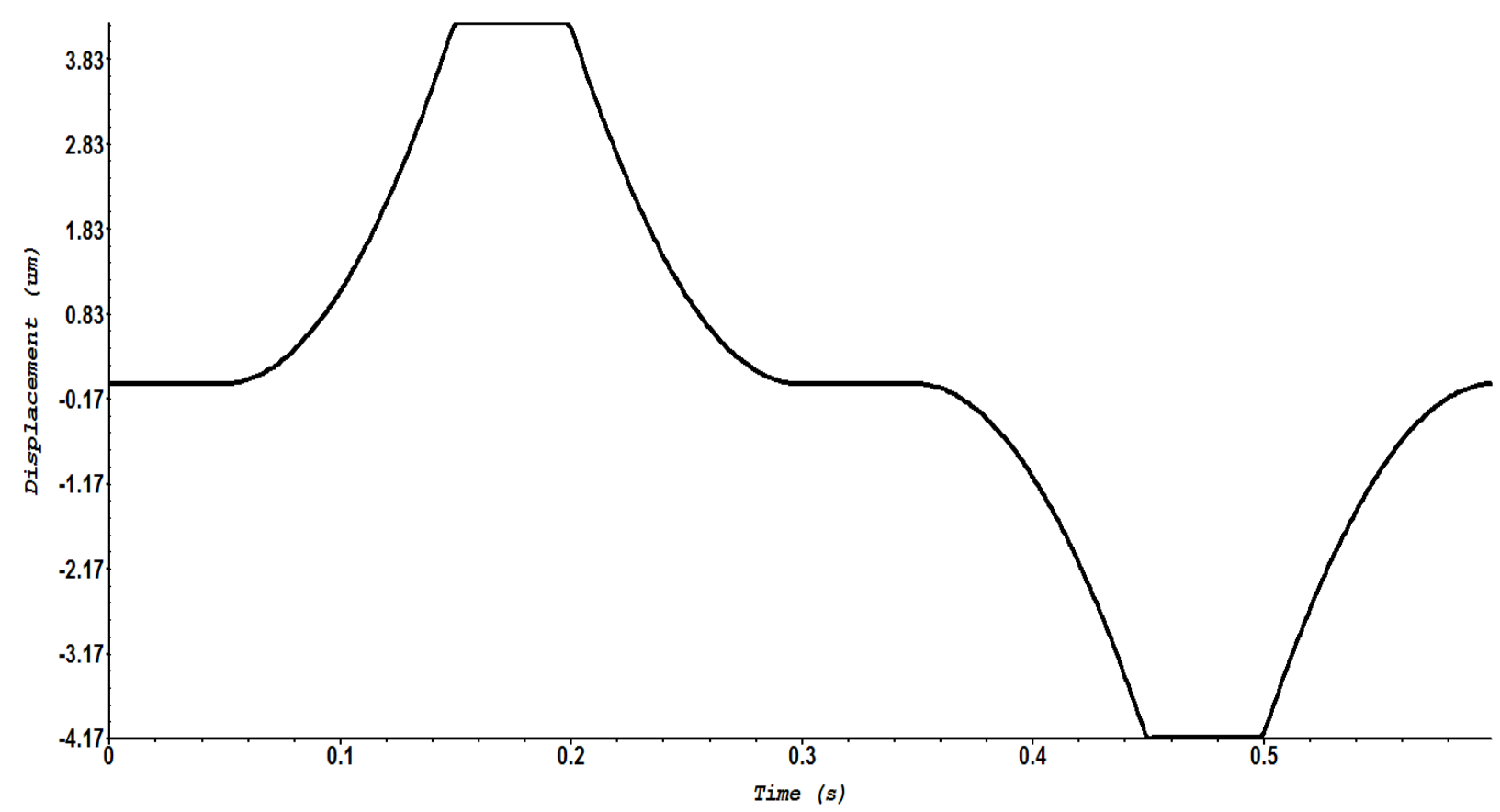

Fig. 6 Displacement vs Time of the 'S' shaped pivot beam

\section{Conclusion}

The ' $\mathrm{S}$ ' shaped RF MEMS seesaw switch uses a reduced set of materials compared to the previous DPDT seesaw switch by using Silicon $(\mathrm{Si})$, Copper $(\mathrm{Cu})$ and Gold $(\mathrm{Au})$ which simplifies the manufacturing process. With the redesign of the pivot the switch attains a greater 'Air-Gap' between the contacts when open-circuited which therefore allows for improved isolation during the off-state.

The air-gap between contacts, in the open position, was increased from $1 \mu \mathrm{m}$ to $2.7 \mu \mathrm{m}$, which increased electrical isolation. 
Using dynamic analysis within Intellisuite and providing simulated activation pulses it was possible to demonstrate the seesaw action ${ }^{9}$; with CST providing isolation and insertion loss results ${ }^{10}$.

The RF MEMS, seesaw switch, with the new 'S' shaped pivot, is a significant improvement over existing designs ${ }^{1,3,5}$.

With the novel 'S' Shaped pivot the von-mises stress at the pivot has been significantly reduced over the original DPDT seesaw switch ${ }^{3}$ allowing the design to take advantage of lower electrostatic pulling forces which in turn reduces the voltage required to pull due to the lower stress on the pivot.

\section{Acknowledgement}

The authors would like to acknowledge Intellisense for their software support and valuable advice on Intellisuite. Also, we would like to acknowledge CST with their support with their software. 


\section{References}

1. J. M. Cabral and A. S. Holmes, "A novel seesaw-type RF MEMS switch," MELECON 2006 - 2006 IEEE Mediterranean Electrotechnical Conference, 288-292 (2006). [doi: 10.1109/MELCON.2006.1653095].

2. H. Kwon et al "Contact materials and reliability for high power RF-MEMS switches," Micro Electro Mechanical Systems, 2007. MEMS. IEEE 20th International Conference on, 231-234 (2007). [doi: 10.1109/memsys.2007.4433055].

3. M. A. Al-Amin, S. Yousef and B. Morris, "RF MEMS DPDT Switch Using Novel Simulated Seesaw Design," International Symposium on Microelectronics 2013(1), 000831-000835 (2013). [doi: 10.1109/ECS.2014.6892558].

4. H. Jaafar, F. L. Nan and N. A. M. Yunus, "Design and simulation of high performance RF MEMS series switch," Micro and Nanoelectronics (RSM), 2011 IEEE Regional Symposium on, 349-353 (2011). [doi:10.1117/1.2338565].

5. J. Kim et al "Variable pivot seesaw actuated RF MEMS switch for reconfigurable system application," Micro Electro Mechanical Systems, 2007. MEMS. IEEE 20th International Conference on, 775-778 (2007). [doi: 10.1109/memsys.2007.4433000].

6. A. S. Revandkar, A. R. Khachane and G. D. Nagare, "Analyzing the effect of metals on the dynamic performance of RF MEMS Switch," Electronics and Communication Systems (ICECS), 2014 International Conference on, 1-5 (2014). [doi: 10.1109/ecs.2014.6892558].

7. G. Schrag, T. Künzig and G. Wachutka, "Modeling reliablity issues in RF MEMS switches," 2013 International Conference on Simulation of Semiconductor Processes and Devices (SISPAD), 432-435 (2013). [doi: 10.1109/sispad.2013.6650667].

8. T. Seki et al, "An RF MEMS switch for 4G Front-Ends," Microwave Symposium Digest (IMS), 2013 IEEE MTT-S International, 1-3 (2013). [doi: 10.1109/mwsym.2013.6697501]. 
9. M. Vakilian, M. Mousavi, B. Bais and B. Y. Majlis, "Optimization of cantilever-based MEMS switch," Semiconductor Electronics (ICSE), 2012 10th IEEE International Conference on, 168-172 (2012). [doi: 10.1109/smelec.2012.6417116].

10. L. Vietzorreck, "EM Modeling of RF MEMS," EuroSime 2006 - 7th International Conference on Thermal, Mechanical and Multiphysics Simulation and Experiments in Micro-Electronics and MicroSystems, 1-4 (2006). [doi: 10.1109/esime.2006.1644057].

Mohammed Abdullah Al-Amin is a final year PhD student at Anglia Ruskin University (ARU). He received his BEng (Hons) in Integrated Engineering (Electronic Route) at ARU in 2009 and is an author of published papers at IMAPS, SDIWC and IAEME. His current research includes RF MEMS switching technologies and simulation techniques. He is a student member is SPIE and IEEE.

Biographies and photographs for the other authors are not available. 


\section{Caption List}

Fig. 1 'S' shaped pivot design.

Fig. 2 RF MEMS 'S' shaped pivot, laid on its side for the manufacturing processes.

Fig. 3 S-Parameters of contacts for the Straight and 'S' Shaped pivot switches.

Fig. 4 Color coded von Mises Stress distribution on the 'S' shaped Pivot.

Fig. 5 Color coded von Mises stress of the original DPDT seesaw switch pivot.

Fig. 6 Displacement vs Time of the 'S' shaped pivot beam.

Table 1 Material information of the 'S' shaped pivot seesaw switch.

Table 2 Material information of RF MEMS DPDT seesaw switch from previous paper ${ }^{3}$.

Table 33 Dimensions of the 'S' shaped RF MEMS pivot DPDT switch and RF MEMS DPDT seesaw switch from previous paper ${ }^{3}$. 